\title{
Collaborative Filtering on Data Streams
}

\author{
Jorge Mario Barajas and Xue Li \\ School of Information Technology and Electrical Engineering, \\ The University of Queensland, Brisbane, Australia \\ s4071254@student.uq.edu.au, xueli@itee.uq.edu.au
}

\begin{abstract}
Collaborate Filtering is one of the most popular recommendation algorithms. Most Collaborative Filtering algorithms work with a static set of data. This paper introduces a novel approach to providing recommendations using Collaborative Filtering when user rating is received over an incoming data stream. In an incoming stream there are massive amounts of data arriving rapidly making it impossible to save all the records for later analysis. By dynamically building a decision tree for every item as data arrive, the incoming data stream is used effectively although an inevitable trade off between accuracy and amount of memory used is introduced. By adding a simple personalization step using a hierarchy of the items, it is possible to improve the predicted ratings made by each decision tree and generate recommendations in real-time. Empirical studies with the dynamically built decision trees show that the personalization step improves the overall predicted accuracy.
\end{abstract}

\section{Introduction}

Nowadays an individual may have access to so many sources of information that is difficult to find the interesting information. The task of a Recommender System 11 is to find interesting items among the vast sea of information. Collaborative Filtering [2] is a recommender system technology that works by building a database of Users-Items with the ratings and then using this database to make recommendations to a user, based on the rating on an item referred to as the Target Item. The whole idea of using users' rating database is to automate the natural social process where people rely on other people's recommendations, this phenomenon is also know as "word of mouth". Collaborative Filtering algorithms can be grouped into two categories: memory-based and model-based [3]. In memory-based algorithms [4, the entire Users-Items database is used to generate a prediction while in model-based approaches [5] a model of user ratings is first developed to make predictions.

One fundamental problem of Collaborative Filtering is managing a big UsersItems database and using it effectively to make predictions about ratings on target items. Any popular online store receives thousands of orders and visits per hour, millions each month. Using all this information to provide quick and quality recommendations using the immense data collected about past users can be challenging. With an incoming data stream it is not possible to build a static 
Users-Items database because there are massive volumes of records arriving continuously at a rapid rate. Generating recommendations over a data stream has the added constraints that the algorithm gets only one look of the data, there is a limit on the number of records it can store, and the recommendations must be made in real-time.

The rest of the document is organized as follows: the next section briefly describes the literature related to collaborative filtering algorithms that aim at giving recommendations in real-time. In section 3 the proposed approach for handling an incoming stream of ratings is described. Section 4 describes the experiments conducted and section 5 finishes with conclusions.

\section{Related Work}

Linden et al [6] proposed the item-to-item collaborative filtering algorithm that scales to massive datasets and provides recommendations in real-time by recording items occurring together. However, the similarities among items are calculated off-line. The off-line batch process is followed by most collaborative filtering algorithms to provide recommendations in real-time, but this produces an outdated model where the quality of the recommendations is low. Online algorithms in Collaborative Filtering [7] are more suitable for handling an incoming data stream since these are fast, incremental and there is no need to store all the previously seen examples. The first online algorithm applied to collaborative filtering was the Weighted Majority Prediction (WMP) [8], Delgado et al [9] extended this approach for multi-valued ratings. Papagelis et al [10] developed a method to incrementally update similarities among users, and Domingos et al [11 proposed VFDT, a system that allows the building of decision trees dynamically to mine data streams.

\section{Proposed Approach}

The goal of data stream processing is to mine patterns, process queries and compute different statistics on data streams in real-time [12. The proposed approach attempts to deal with the prediction problem of Collaborative Filtering when the ratings are received over a continuous data stream. The prediction problem in Collaborative Filtering over a data stream is defined as follows. Having a list of items $I$ and a set of online users $U$, an incoming stream $S$ of opinions $\left\langle U_{i},\left\{I_{j}, \ldots, I_{k}\right\},\left\{O_{j}, \ldots, O_{k}\right\}\right\rangle$ is received, where $U_{i}$ identifies the $i$-th online user, $\left\{I_{j}, \ldots, I_{k}\right\} \subset I$ is the set of items rated by $U_{i}$, and $\left\{O_{j}, \ldots, O_{k}\right\}$ are the opinions of user $U_{i}$ on items $\left\{I_{j}, \ldots, I_{k}\right\}$. The task is to predict in real-time, the opinion $O_{b}$ of the online user $U_{i}$ on a target item $I_{b}$ where $I_{b} \notin\left\{I_{j}, \ldots, I_{k}\right\}$.

The main idea of the proposed approach is to build a decision tree for every item by assuming they are all related to each other, using a very fast algorithm to handle the rapid incoming stream of ratings effectively and then personalizing the predicted rating made by the decision tree by scaling it up or down depending on the hierarchy of items liked by the user. The scaling of the decision trees predicted 
value with the items' hierarchy deals with the inevitable hit in accuracy that is introduced when building the decision trees over the incoming stream. The next sections describe the proposed approach in detail.

\subsection{Building Decision Trees Dynamically}

Observing the layout of the Users-Ratings database, the prediction of the rating for a target item by the active user can be seen as a classification problem [13] where an attribute value is found instead of trying to come up with a number between the ratings's range. The classification problem view can be applied easily to both scaled or binary ratings. With 0-5 rating values, each item has 6 classes and with binary ratings only 2 ('Disliked' and 'Liked'). Having $N$ items, the classification problem involves $N-1$ attributes (the rest of the items in the database) and the class is the target's item rating.

For every item it is necessary to find other items that have stronger predictive capacity so they can be used in the prediction of the item's rating. If someone purchases a cereal he would eventually need milk, or if someone purchases a lamp he would need a bulb. The idea is to find these items that are strongly associated with the target item and use their ratings to predict the target's rating. Decision trees are useful for this task, but with an incoming data stream they need to be built dynamically since it is not possible to load all the examples into memory. The VFDT learning system [11] allows the building of a decision tree on a data stream by considering a small subset of cases to find the best attribute to make a split decision using information gain. By using VFDT, it is possible to build a decision tree for every item in the database when the ratings are received over a data stream as shown in figure 1. Nevertheless, the dynamic building of decision trees for each item brings a trade-off between the accuracy and the amount of memory used to store the records statistics.

\begin{tabular}{|c|c|c|c|c|c|c|c|}
\hline Time & Coke & Pepsi & Wine & Lettuce & Tomato & Onion & Broccoli \\
\hline 10:51:11 & '1' & '4' & ${ }^{\prime}{ }^{\prime}$ & '2' & '2' & ' ' & '5' \\
\hline 10:51:12 & 3 & 2 & 2 & $?$ & 11 & ${ }^{5}$ & 2 \\
\hline 10:51:13 & 1 ' & $?$ & '1' & 4 & 4 & $?$ & $2^{\prime}$ \\
\hline $10: 51: 14$ & 1 ' & ? & 3 & 2 & 3 & ? & 2 \\
\hline $10: 51: 15$ & 3 & '1' & ? & 2 & ? & 3 & ? \\
\hline 10:51:16 & '1' & $?$ & '1' & 3 & 2 & ? & 4 \\
\hline $10: 51: 17$ & 4 & 4 & $?$ & 3 & $3^{\prime}$ & 3 & $?$ \\
\hline
\end{tabular}

Fig. 1. Building decision trees for every item in the database dynamically

\subsection{The Hierarchy of Items}

Once the prediction of a rating for an active user has been made, using the dynamically built decision trees, the predicted rating can be improved. Each decision tree 
provides a predicted rating that has been proven to be true with the general statistical properties of the past examples, no true personalization of the results has been done. An accuracy hit has also been added to the decision trees by building them dynamically. There is still the issue of having to operate on a data stream where there is little or no information at all about past users to use in this personalization step. Here is where some information known or derived about the items can be used online to improve the prediction made by the decision tree.

The hierarchy of items [14] can be used to make certain assumptions about the users' interests. One possible way to start understanding a user's interests is when he starts pointing out some of the items that will be interesting to him. If a hierarchy of the items liked by the user is built, this hierarchy can be used to look for items that belong to categories contained in this hierarchy and provide recommendations for that user. If an item was predicted as disliked by the active user by the item's decision tree and this item belongs to one of the categories contained in the active user's hierarchy of liked items, it is possible to argue that the decision tree is not completely applicable to this user and the rating should be scaled up.

There are some important aspects to take into account when using the hierarchy of items to scale up or down the predicted rating given by the dynamically built decision trees. Scaling up all the ratings of items belonging to the built hierarchy that were marked as disliked by the decision tree can be too aggressive. A user might go to purchase groceries at a supermarket where the taxonomy of items is varied and complex, his list of groceries will include many items of many taxonomic categories but he will probably only be interested in a few products that belong to his favorite category (e.g. Cereals) and he usually purchases a lot of items belonging to this single category. Is in this case where it makes sense to scale up all the ratings of the items predicted as disliked belonging to the user's favorite category or set of favorite categories so he can receive recommendations about items he hasn't seen but belong to the favorite category of the active user.

\section{Experiments and Results}

A series of experiments were conducted to examine the prediction ability of the decision trees built dynamically for each item and the improving capability of using a hierarchy of items. In particular, for the decision trees it was important to first examine which attribute values definition had a better performance for building dynamically the decision trees and how these performed when exposed to different number of attributes. VFDT [1] was the algorithm used to build the decision trees for each item. Finally, the improving ability of using a hierarchical taxonomy of items to change the predictions given by each decision tree was evaluated.

\subsection{Experiments Setup}

The proposed approach was evaluated with EachMovie1. EachMovie is a dataset of movie ratings made publicly available by Digital Equipment Corporation (DEC)

$\overline{{ }^{1} \text { http://research.compaq.com/SRC/eachmovie/ }}$ 
for Collaborative Filtering research. The dataset contains the numeric ratings for 1628 movies given by 72916 users. Each numeric rating is a score from 0.0 to 1.0 with 0.2 spaces that maps linearly to the zero-to-five star rating were a five star rating stands for a 'liked it very much' and a 0 star rating for a 'Didn't or won't like it'. Each vote is accompanied by a time stamp. For the experiments a subset of 500 movies with the greatest number of votes was first extracted. The entries were ordered by the time stamp to simulate an incoming data stream. The evaluation metrics used were the Mean Absolute Error (MAE) and the Mean Squared Error (MSE) which are shown in equations 1 and 2 and where $p_{i}$ and $q_{i}$ is the predictedrating pair for item $i$, and $N$ is the total number of items. These two metrics were measured for the whole database and for each movie.

$$
\begin{gathered}
M A E=\frac{\sum_{i=0}^{N}\left|p_{i}-q_{i}\right|}{N} \\
M S E=\frac{\sum_{i=0}^{N}\left|p_{i}-r_{i}\right|^{2}}{N}
\end{gathered}
$$

\subsection{Experiment 1: Decision Trees'Attributes Evaluation}

In our first experiment, the goal was to find out which was the best way to build the decision trees for every item in terms of the definition of the attributes' values. The evaluation metrics were calculated with the distance between the predicted rating class and the real rating given by the user. Only 50 Movies with the greatest number of votes were used. VFDT's parameters were initialized to nominal values: $\tau=0.055, \delta=0.0000001$ and $n_{\min }=250$. The results are shown in figure 2 .

The decision trees built with 7 cardinal valued attributes outperformed the others built with different attribute values. In this setting, every unknown rating
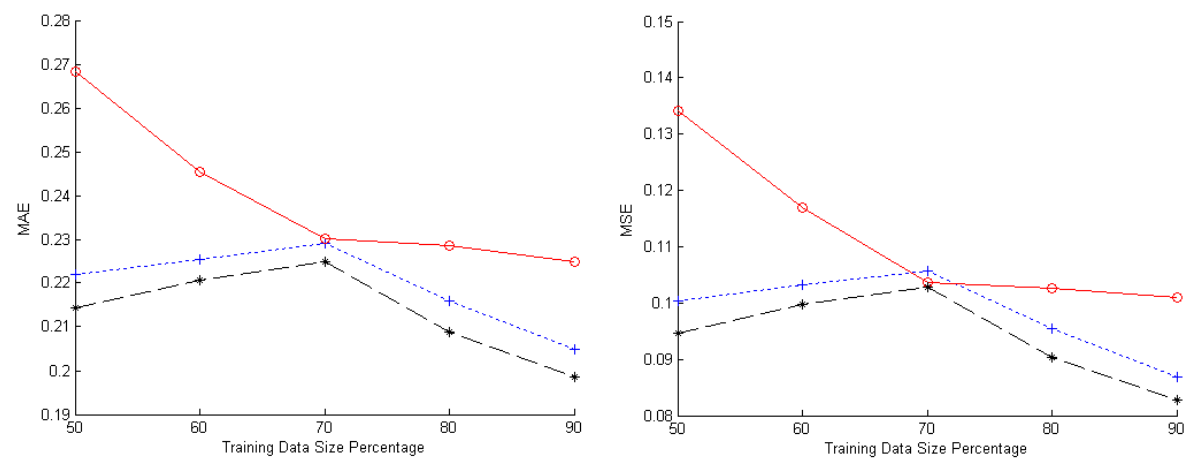

$$
\begin{array}{|l}
-+ \text { Ordinal Attributes with Missing Values } \\
-6 \text { Cardinal Attributes with Missing Values } \\
+7 \text { Cardinal Attributes with no Missing Values } \\
\hline
\end{array}
$$

Fig. 2. Dynamically built decision trees performance with different types of attributes 
was assigned to a new 'Undefined' class so there weren't any missing values. The decision trees built with continuous attributes also performed well compared with the decision trees built with 6 valued attributes and missing values.

\subsection{Experiment 2: Number of Attributes for Decision Trees}

To see how the decision trees behaved when exposed to more attributes and more data sparsity, movies with the greatest number of votes were added from the unused subset. These experiments used 80 percent of the dataset to build the decision trees, and the other 20 percent was used to test them measuring the MAE and MSE as in the first experiment. VFDT's parameters were set to $\tau=0.1, \delta=0.0000001$ and $n_{\min }=250$. Results are shown in table 1. The decision trees performed relatively stable when exposed to more data. The more attributes are available, the lower the MAE for the best performing decision tree. This can be explained by the fact that for building each decision tree there are more items so there is a bigger chance of finding items with stronger predictive capacity and the target item's predicted rating is more accurate. On the other hand, the worst performing decision trees decreased their accuracy with more attributes because some items didn't have enough examples to build an accurate decision tree.

\subsection{Experiment 3: Using the Items' Hierarchy}

In order to improve the decision's trees results using the item's hierarchy and face the accuracy trade off by dynamically building the decision trees, the genre classification about each movie was used. In the EachMovie dataset, each movie belongs to one or more genres, to deal with this, the set of genres of each movie were treated as one category; that is, if a movie belonged to the genres action and drama, a category called 'Action and Drama' was created and all the movies belonging to only these two genres were added to the category. The settings of experiment 4.3 were used and a hierarchy of items was built with the items liked by the user: those that had a rating greater or equal to 4 stars. If the target item didn't belong to any of the genres contained in the hierarchy of items, the decision trees' predicted rating were decreased by one if it was liked, if the movie

Table 1. Errors of the dynamically built decision trees

\begin{tabular}{lllllll}
\hline Movies & MAE & MSE & $\begin{array}{l}\text { MAE } \\
\text { Best } \\
\text { DT }\end{array}$ & $\begin{array}{l}\text { MAE } \\
\text { Worst } \\
\text { DT }\end{array}$ & $\begin{array}{l}\text { MSE } \\
\text { Best } \\
\text { DT }\end{array}$ & $\begin{array}{l}\text { MSE } \\
\text { Worst } \\
\text { DT }\end{array}$ \\
\hline 100 & 0.2062 & 0.0896 & 0.1089 & 0.5401 & 0.0319 & 0.4130 \\
200 & 0.2166 & 0.0984 & 0.0676 & 0.5401 & 0.0370 & 0.4130 \\
300 & 0.2224 & 0.1043 & 0.0676 & 0.5581 & 0.0291 & 0.4327 \\
400 & 0.2238 & 0.1062 & 0.0676 & 0.5687 & 0.0336 & 0.4327 \\
500 & 0.2287 & 0.1111 & 0.0676 & 0.7526 & 0.0336 & 0.6071 \\
\hline
\end{tabular}


belonged to the most popular set of genres in the hierarchy of liked items by the user, the decision trees' predicted rating was increased by one if it was disliked (less than or equal to '3'), otherwise the decision trees' predicted rating was left unchanged. Results are shown in table 2 .

Table 2. Hierarchy Personalization Errors

\begin{tabular}{lllllll}
\hline Movies & MAE & MSE & $\begin{array}{l}\text { MAE } \\
\text { Best } \\
\text { DT }\end{array}$ & $\begin{array}{l}\text { MAE } \\
\text { Worst } \\
\text { DT }\end{array}$ & $\begin{array}{l}\text { MSE } \\
\text { Best } \\
\text { DT }\end{array}$ & $\begin{array}{l}\text { MSE } \\
\text { Worst } \\
\text { DT }\end{array}$ \\
\hline 100 & 0.2039 & 0.0818 & 0.1074 & 0.5051 & 0.0296 & 0.3608 \\
200 & 0.2127 & 0.0891 & 0.0692 & 0.4801 & 0.0316 & 0.3224 \\
300 & 0.2185 & 0.0946 & 0.0668 & 0.5437 & 0.0302 & 0.3866 \\
400 & 0.2203 & 0.0965 & 0.0721 & 0.5424 & 0.0331 & 0.3853 \\
500 & 0.2242 & 0.1002 & 0.0709 & 0.7522 & 0.0331 & 0.6065 \\
\hline
\end{tabular}

The use of the liked items hierarchy per user effectively improved the decision trees' results. Each decision tree was built dynamically so there was an inevitable tradeoff in the accuracy of the predicted rating and the amount of memory used. As we can see from table 4 that the MSE was lowered, meaning that the big errors produced by the decision trees were reduced. In all cases the performance of prediction, with the worst-case errors, is improved.

\section{Conclusions}

This paper introduced a novel approach to providing recommendations with Collaborative Filtering over an incoming data stream containing user rating over items. By dynamically building decision trees for every item, it is possible to deal with an incoming data stream and use all received data to generate recommendations. A hierarchy of items was used to improve the personalization of each generated decision tree. Moreover, by using different types of hierarchies of items, the same set of decision trees may behave differently for the different mappings between the items and their categories. So, different item hierarchies can lead to different predictions. For example a hierarchy of items might be a taxonomy that focuses on understanding of user profile. Then the recommendation will be based on user tastes. While another hierarchy could group popular and unpopular items that will lead to some novel recommendations.

The results of our experiments have shown that our proposed approach of building the multiple decision trees on the fly for the real-time recommendation is effective and efficient.

\section{Acknowledgements}

This work is partially founded by the Australian ARC Large Grant DP0558879. 


\section{References}

1. Resnick, P., Varian, H.R.: Recommender systems. Commun. ACM 40 (1997) 56-58

2. Goldberg, D., Nichols, D., Oki, B.M., Terry, D.: Using collaborative filtering to weave an information tapestry. Commun. ACM 35 (1992) 61-70

3. Breese, J.S., Heckerman, D., Kadie, C.: Empirical analysis of predictive algorithms for collaborative filtering. In: Fourteenth Annual Conference on Uncertainty in Artificial Intelligence. (1998) 43-52

4. Sarwar, B.M., Karypis, G., Konstan, J.A., Reidl, J.: Item-based collaborative filtering recommendation algorithms. In: World Wide Web. (2001) 285-295

5. Hofmann, T.: Collaborative filtering via gaussian probabilistic latent semantic analysis. In: SIGIR '03: Proceedings of the 26th annual international ACM SIGIR conference on Research and development in informaion retrieval, New York, NY, USA, ACM Press (2003) 259-266

6. Linden, G., Smith, B., York, J.: Industry report: Amazon.com recommendations: Item-to-item collaborative filtering. IEEE Distributed Systems Online 4 (2003)

7. Calderón-Benavides, M.L., González-Caro, C.N., de J. Pérez-Alcázar, J., GarcíaDíaz, J.C., Delgado, J.: A comparison of several predictive algorithms for collaborative filtering on multi-valued ratings. In: SAC '04: Proceedings of the 2004 ACM symposium on Applied computing, New York, NY, USA, ACM Press (2004) 1033-1039

8. Nakamura, A., Abe, N.: Collaborative filtering using weighted majority prediction algorithms. In: ICML '98: Proceedings of the Fifteenth International Conference on Machine Learning, San Francisco, CA, USA, Morgan Kaufmann Publishers Inc. (1998) 395-403

9. Delgado, J., Ishii, N.: Memory-based weighted-majority prediction for recommender systems. In: Proceedings of the ACM SIGIR-99. (1999)

10. Papagelis, M., Rousidis, I., Plexousakis, D., Theoharopoulos, E.: Incremental collaborative filtering for highly-scalable recommendation algorithms. In: International Symposium on Methodologies of Intelligent Systems (ISMIS'05). (2005)

11. Domingos, P., Hulten, G.: Mining high-speed data streams. In: Knowledge Discovery and Data Mining. (2000) 71-80

12. Garofalakis, M.N., Gehrke, J.: Querying and mining data streams: You only get one look. In: VLDB. (2002)

13. Basu, C., Hirsh, H., Cohen, W.W.: Recommendation as classification: Using social and content-based information in recommendation. In: AAAI/IAAI. (1998) 714720

14. Ganesan, P., Garcia-Molina, H., Widom, J.: Exploiting hierarchical domain structure to compute similarity. ACM Trans. Inf. Syst. 21 (2003) 64-93 